\title{
Eficiencia escolar y diferencias socioeconómicas: a propósito de los resultados de las pruebas de medición de la calidad de la educación en Chile.
}

\author{
Sebastián Donoso Díaz \\ Universidad de Talca \\ Gustavo Hawes Barrios \\ Universidad de Talca
}

Correspondência:

Sebastián Donoso Díaz

Instituto de Investigación y

Desarrollo Educacional

Universidad de Talca

Casilla 747, Talca - Chile

e-mail: sdonoso@utalca.cl

ghawes@utalca.cl

\begin{abstract}
Resumo
El artículo analiza para el sistema educacional chileno, los resultados obtenidos en las principales asignaturas (matemática y lenguaje) en el Sistema de Medición de la Calidad de la Educación (Simce) por los $8^{\text {os }}$ años en el año 2000.

Se revisan los resultados primero en sus aspectos generales y luego se comparan dichos resultados, controlados por nivel socioeconómico de los establecimientos, efectuándose diversas agrupaciones de información que confirman que la variable socioeconómica es el factor determinante en los resultados, y que la dependencia de la escuela (si es privada, con aportes del estado o plenamente estatal) pierde fuerza, y que controlados por el valor de las mensualidades (costo directo) la eficiencia de los resultados favorece a los establecimientos que atienden población más vulnerable.

En este marco destacan dos aspectos: los establecimientos municipales son eficientes para el sector más pobre de la población, y segundo, en los niveles socioeconómicos en los que hay plena competencia (el mercado funciona) la dependencia del establecimiento es secundaria, siendo el nivel socioeconómico el factor explicativo dominante.

Comparando los establecimientos por grupo socioeconómico y dependencia del éste se omite un factor de inequidad que tiene incidencia sobre los resultados escolares: el equipamiento e infraestructura escolar, partiendo de una situación ficticia (igualdad de procesos básicos) la que favorece a los establecimientos mejor dotados, que son los que incorporan financiamiento de los padres. Ello pone en duda que las diferencias "intragrupo socioeconómico" se deben a las variables de "gestión escolar", la evidencia es contundente al incorporar el nivel socioeconómico como variable explicativa.
\end{abstract}

\section{Palavras-chave \\ Desigualdades sociales y educativas - Eficiencia escolar — Resulta- dos escolares - Resultados según tipo de establecimiento.}




\title{
School efficiency and socioeconomic differences: on the results of assessment exams of the Quality of Education in Chile
}

Sebastián Donoso Díaz

University of Talca

Gustavo Hawes Barrios

University of Talca

Correspondence:

\begin{abstract}
The article analyzes the results verified in the main disciplines (Mathematics and Spanish) in the Chilean Quality of Education Assessment System (SIMCE) in 2000 for the $8^{\text {th }}$ grade of the elementary school.

Firstly, the general aspects of the results are examined, and then they are compared on the basis of the socioeconomic level of the schools. The various groups of information collected confirm that the socioeconomic variable is the determinant factor in the results, and that the school affiliation (private, public or state funded) loses importance. When checked against the cost of school fees (direct cost), the efficiency of results favors the schools that serve the more vulnerable population.

In that respect, two aspects are of relevance: first, the municipal schools are efficient to the poorer layers of the population; second, at the socioeconomic levels where there is total competence (the market works), the dependence on the school is secondary, the socioeconomic level being the dominant explaining factor.

Comparison of schools by socioeconomic group and affiliation eliminates an inequality factor that has effect on the performance results: the school equipment and infrastructure, if considered in a fictitious situation (equality of basic processes), favor the better equipped schools, which incorporate funding from the parents. This casts doubt on the assertion that differences intra-socioeconomic group are due to the school management variables; the evidence is compelling when the socioeconomic level is incorporated as an explaining variable.
\end{abstract}

\section{Keywords}

Social and education inequalities - School efficiency School results. 


\section{El Sistema de medición de la calidad de la educación en Chile}

Desde su gestación en el año 1988, el Simce $^{1}$ se basa en una prueba anual que se aplica a escala nacional - alternadamente a los $4^{\circ}$ u $8^{\circ}$ básico y $2^{\circ}$ medio - por igual a todos los alumnos que cursan el nivel. Su administración se realiza de manera externa al establecimiento educacional, pero no al sistema mismo.

Su objetivo principal "es generar indicadores confiables que sirvan para orientar todo tipo de acciones y programas de mejoramiento de la calidad de la enseñanza" (Mineduc, 2001). Los puntajes del Simce permiten conocer el desempeño en diferentes asignaturas y dimensiones de cada establecimiento educacional, comparando los resultados con años anteriores y también respecto de otros establecimientos con características socioeconómicas afines. De igual forma, permite contrastar el desempeño de cada curso en un mismo establecimiento.

El Simce 2000 evaluó los objetivos generales y específicos del Programa de Estudios para 8o básico del Decreto 4002 de 1980, esto es, el currículo "no reformado". Tratándose de una prueba basada en el currículo, este formato la hace más independiente de las influencias del capital cultural heredado.

La metodología de evaluación usada en el Simce es la misma de la mayoría de las mediciones internacionales de este tipo. Emplea una escala de puntajes relativa. No define previamente valores mínimos o máximos establecidos, pero los puntajes se estandarizan con promedio 250 y desviación estándar de 50 puntos. De esta manera, se asume que los puntajes por sobre ese valor reflejan aprendizaje superior al promedio y los menores, resultados inferiores, escalándose a los establecimientos en función del promedio obtenido por sus estudiantes en cada una de las pruebas.

Como todo sistema de evaluación, tiene un margen de incertidumbre. Al realizarse las comparaciones entre los puntajes de los distintos grupos, las variaciones $^{2}$ deben ser mayores a un valor mínimo, de esta forma se asume que reflejan diferencias reales en los logros de aprendizaje. Este es punto importante de tener presente toda vez que un porcentaje no menor de las escuelas tienen tamaños inferiores a los indicados.

Por otra parte, las comparaciones con los resultados del año 1997 (medición anterior de $8^{\circ}$ básico), si bien la escala de puntajes utilizada era diferente, se realizaron mediante la inclusión en la última prueba de un cierto número de preguntas que ya habían formado parte de las pruebas de ese año. Los cálculos de las comparaciones, tanto en el ámbito nacional como del establecimiento, se hicieron a partir de los resultados de este conjunto de preguntas. Desde el punto de vista conceptual, el cambio del enfoque de medición entre la prueba de 1997 y la del 2000 es relevante e implica pasar de medir el promedio de aprendizaje de los alumnos de una escuela, a estimar los alumnos de una escuela que demuestran lograr un cierto aprendizaje.

En el presente artículo analizamos la relación de los resultados en el Simce con la estructura socioeconómica de los establecimientos y su régimen de dependencia a fin de determinar hasta qué punto las diferencias sociales explican los resultados del Simce. Finalmente, se evalúa, a grandes líneas el valor de las puntuaciones Simce en función de las diversas estructuras de dependencia.

1. Sistema de Medición de la Calidad de la Educación. Considera pruebas de Lenguaje, Matemática, Ciencias Naturales y Ciencias Sociales; además, una encuesta con padres, profesores y otros elementos. Sin embargo las pruebas a los estudiantes son el eje del tema.

2. Las comparaciones tienen mayor validez cuando se refieren a grupos iguales o superiores a 30 alumnos (tamaño típico de un curso). En general, al comparar grupos de menor tamaño, las variaciones deben ser mayores para ser consideradas significativas 
La clasificación socioeconómica de los establecimientos educacionales

El Simce asume que los resultados del proceso educativo se ven fuertemente influenciados por el nivel socioeconómico de las familias y por ende, del establecimiento escolar. Este considerando tiene sólidos fundamentos en múltiples trabajos desde Coleman (1969, 1990) y Jenks (1972), hasta nuestros días. Esta influencia tiene a su vez impactos culturales, lo que explica la fuerza sinérgica que revisten las variables socioeconómicas sobre el resto de las variables escolares, incluyendo las de gestión de los establecimientos (Alvariño et al., 2001).

De igual forma, una clasificación socioeconómica acertada facilita la comparabilidad de los establecimientos afines. De esta manera, un desempeño mejor que otro permite estimar con una buena probabilidad, que las diferencias se deben a lo que sucede "dentro del establecimiento" más que fuera de él.

Los establecimientos se clasificaron según las condiciones sociales y económicas de las familias que atienden. Se definen cinco grupos utilizando variables que han mostrado significación para estos efectos: escolaridad del padre y de la madre, ingresos económicos promedio del Hogar e Índice de Vulnerabilidad promedio del establecimiento. ${ }^{3}$ De acuerdo con lo señalado, en la medición del año 2000 la situación de los establecimientos por estrato socioeconómico es la siguiente:

\section{Los resultados en función de la estructura de clasificación socioeconómica de los establecimientos}

En primera instancia, en el ámbito general puede señalarse que los promedios nacionales para el país en su conjunto revelan mejoramientos significativos tanto en Historia y Geografía (+6 puntos) como en Ciencias Naturales (+10 puntos), siendo menos pronunciados para el nivel socioeconómico más bajo.

Los promedios nacionales suelen ser estables, de forma que ambos casos reflejan avances de consideración. Las variaciones observadas en Matemática (0 puntos) y en Castellano (-3 puntos) no alcanzan a ser considerados relevantes, conforme a la metodología de comparación utilizada (Mineduc, 2001).

El análisis por grupo socioeconómico muestra que los resultados mejoran a medida que aumenta el nivel socioeconómico. En consecuencia, inicialmente podríamos decir que la pobreza es factor asociado a los bajos rendimientos, situación que reviste mayor incidencia que la

3. Una discusión más detallada de estas variables podría llevarnos a la consideración que unas comprenden a otras, y por ende se encuentran fuertemente asociadas (multicolineales). Por ejemplo escolaridad de los padres tiene esa característica, siendo la educación de la madre más decisiva a la hora de explicar los rendimientos escolares. Lo mismo al analizar el nivel de vulnerabilidad de un establecimiento, que considera la escolaridad de los padres como un constituyente. De todas formas esta situación puede generar sobreestimaciones dentro de determinados rangos.

Tabla $n^{\circ}$ 1. Distribución de los valores de clasificación socioeconómica por Estrato

\begin{tabular}{|c|c|c|c|c|c|c|}
\hline \multirow{2}{*}{$\begin{array}{c}\text { Grupo } \\
\text { socio-económico }\end{array}$} & \multicolumn{2}{|c|}{ Alumnos } & \multicolumn{2}{|c|}{$\begin{array}{l}N^{\circ} \text { años promedio de } \\
\text { escolaridad }\end{array}$} & \multirow{2}{*}{$\begin{array}{l}\text { Promedio de } \\
\text { ingreso del hogar } \\
\text { (\$ an้̂o 2000) }\end{array}$} & \multirow[t]{2}{*}{$\begin{array}{l}\text { Prom IVE' } 2000 \\
\text { / JUNAEB }\end{array}$} \\
\hline & Múmero & $\%$ & Madre & Padre & & \\
\hline$A($ Bajo) & 23,052 & $9 \%$ & 6 & 7 & $\$ 92,000$ & $70 \%$ \\
\hline B (Medio bajo) & 79,395 & $32 \%$ & 8 & 8 & $\$ 130.000$ & $44 \%$ \\
\hline C (Medio) & 88.647 & $35 \%$ & 10 & 11 & $\$ 198,000$ & $23 \%$ \\
\hline D (Medo alio) & 41,920 & $17 \%$ & 13 & 13 & $\$ 409,000$ & $7 \%$ \\
\hline$E$ (Alto) & 18,604 & $7 \%$ & 16 & 17 & $\$ 1,163,000$ & $0 \%$ \\
\hline
\end{tabular}


dependencia del establecimiento, afirmación que constituye una hipótesis central de trabajo.

Para el grupo socioeconómico A, que concentra los establecimientos de familias de menor nivel socioeconómico (9\% de la población escolar), los resultados obtenidos por las escuelas municipales son entre $7 \mathrm{y}$ 10 puntos superiores al particular subvencionados, lo que puede interpretarse como que el sector municipal atiende mejor a este grupo social.

Para el grupo socioeconómico siguiente (medio bajo), que involucra al $32 \%$ de los estudiantes, prácticamente no existen diferencias importantes en resultados entre ambas estructuras de depen-dencia. Esta situación se mantiene en el nivel medio-medio, con una tendencia positiva hacia los establecimientos particulares subvencionados.

Para el grupo D, único en que coexisten los tres tipos de dependencia (municipales, particulares subvencionados y particulares pagados), los resultados son re- lativamente similares para los tres tipos de establecimientos. Los particulares subvencionados son quienes obtienen un puntaje ligeramente inferior en todas las asignaturas. Los particulares pagados aventajan a los municipales por entre 2 y 6 puntos en tres asignaturas.

En el Grupo E, sólo hay particulares subvencionados y pagados, y los resultados favorecen tendencial pero no significativamente a los subvencionados.

Como sintesis de esta primera estructura de análisis, puede señalarse que el nivel socioeconómico promedio de las familias explica en gran medida los resultados escolares alcanzados. En este marco destacan dos aspectos: primero que para el sector más pobre de la población los establecimientos municipales son eficientes, y en segundo lugar, que en los niveles socioeconómicos en que hay plena com-petencia (el mercado funciona) la dependencia del establecimiento es secundaria, siendo el nivel socioeconómico el factor explicativo dominante.

Tabla $n^{\circ}$ 2. Resultados Generales del SIMCE por estructura socioeconómica y pruebas

\begin{tabular}{|c|c|c|c|c|c|c|c|c|c|c|}
\hline \multirow{3}{*}{$\begin{array}{c}\text { Totales } \\
\text { Nacionales }\end{array}$} & \multirow{2}{*}{$\begin{array}{c}\mathrm{N}^{3} \\
\text { Ecuelas }\end{array}$} & \multirow[t]{2}{*}{$N^{*}$ Alumnos } & \multicolumn{2}{|c|}{ Matemática } & \multicolumn{2}{|c|}{ Castellano } & \multicolumn{2}{|c|}{$\begin{array}{l}\text { Historia y } \\
\text { Geografia }\end{array}$} & \multicolumn{2}{|c|}{ Cs. Naturales } \\
\hline & & & Prom & Var & Pram & $\mathrm{Var}$ & Prom & Var & Pronil & Var \\
\hline & 5.044 & 251.618 & 250 & 0 & 250 & $-3 \bullet$ & 250 & +64 & 250 & $+10 *$ \\
\hline A Bajo & 1.160 & 23.052 & 230 & $-1 \cdot$ & 228 & $-2 \cdot$ & 228 & $+3 \cdot$ & 233 & +2 \\
\hline B Medio Bajo & 1.538 & 79.395 & 232 & $-1 \cdot$ & 233 & $-3 \cdot$ & 233 & $+4 \cdot$ & 234 & $+9 \bullet$ \\
\hline CMedio & 1.230 & 88.647 & 248 & $+2 \cdot$ & 249 & $-2 \bullet$ & 249 & $+9+$ & 248 & $+14 *$ \\
\hline D Medio Alto & 653 & 41.920 & 277 & $+2 \cdot$ & 276 & $-3 \bullet$ & 275 & $+7+$ & 273 & $+12+$ \\
\hline EAto & 463 & 18.604 & 302 & +2 & 297 & $-4 \cdot$ & 296 & $+10 \bullet$ & 297 & $+12 \bullet$ \\
\hline
\end{tabular}

Tabla $n^{\circ}$ 3. Resultados generales del SIMCE por estructura socioeconómica, dependencia y prueba

\begin{tabular}{|c|c|c|c|c|c|c|c|c|c|c|c|c|}
\hline \multirow{2}{*}{ NSE } & \multicolumn{3}{|c|}{ Matemática } & \multicolumn{3}{|c|}{ Castellano } & \multicolumn{3}{|c|}{ Historia y Geografia } & \multicolumn{3}{|c|}{ Ciencias Naturales } \\
\hline & Munic & $P S$ & $P P$ & Munic & PS & $\rho p$ & Munic & PS & $P P$ & Munic & $P S$ & $\overline{\phi \rho}$ \\
\hline A Bajo & 231 & 221 & - & 230 & 221 & $\ldots$ & 230 & 221 & - & 234 & 222 & - \\
\hline B Nedio Bajo & 232 & 233 & $\ldots$ & 232 & 234 & $*$ & 232 & 234 & $*$ & 234 & 235 & $*$ \\
\hline CNedio & 245 & 251 & $\ldots$ & 246 & 252 & $\ldots$ & 246 & 253 & - & 245 & 251 & $=$ \\
\hline D Medio Alto & 280 & 275 & 269 & 278 & 275 & 280 & 276 & 284 & 282 & 272 & 272 & 280 \\
\hline E Alto & - & 303 & 302 & - & 297 & 297 & $\ldots$ & 295 & 296 & - & 299 & 291 \\
\hline
\end{tabular}


Discusión de algunos supuestos e hipótesis explicativas de los resultados

Un supuesto fundamental del Simce (que es una hipótesis de trabajo) es la comparabilidad de los resultados registrados en el proceso. Esta condición está en la base misma del sistema y sin ella no se podría operar. Ciertamente al fundarse en la medición del currículo básico común a todos los establecimientos escolares, es posible realizar esta operación, pero no es suficiente en sí misma para que los resultados tengan equivalencia (validez) sustantiva, dado que no da cuenta de variables contextuales que son decisivas en relación a los niveles de logro de los estudiantes.

Por ello - creemos - las condiciones de comparabilidad deben ser discutidas más en detalle, toda vez que éstas se han definido a partir de la variable socioeconómica promedio del establecimiento, con propiedades de medición ordinales, con cinco tramos, derivados de la conjugación de los valores antes expuestos (Tabla $n^{\circ} 1$ ).

Lo más relevante en este caso sería conocer el peso estadístico de cada grupo en función de los factores socioeconómicos y en función de ello determinar el valor estadístico o distancia que deberian tener entre sí. De esta forma, las diferencias establecidas representarian un factor real de comparación a partir del cual podrían reducirse los resultados a unidades iguales (escala) y así poder determinar la estructura de los resultados y su significación. ${ }^{4}$ En razón de ello, los resultados actuales ocultan una serie de situaciones que no es posible determinar más que a nivel macro y que en lo fundamental subestiman el efecto de las variables socioeconómicas. ${ }^{5}$

En función de lo expuesto, categorizar los grupos socioeconómicos básicamente por las diferencias familiares (escolaridad de los padres, ingreso familiar e índice de vulnerabilidad del establecimiento) es relevante pero ciertamente insuficiente pues implícitamente asume (más allá del factor vulnerabilidad de la Escuela) que el proceso escolar es idéntico, al menos en recursos, equipamiento e infraestructura. Este segundo supuesto es muy débil, dado que es sabido que el factor recursos pedagógicos también está asociado al nivel socioeconómico de las familias de un establecimiento, de manera que fortalece las diferencias generadas por la clasificación anterior o, tratándose de escuelas municipales, a la capacidad económica de las diferentes comunas.

En la actualidad, en el debate público de los recursos asignados a educación se menciona el gran incremento del gasto en educación efectuado por el Ministerio de Educación y las enormes cifras que se destinan a la educación pública. Siendo ello verdadero, también deberá concordarse que pese a esta situación, la disponibilidad de equipamiento e infraestructura del sector público respecto del privado con financiamiento compartido y pagado es aún muy desfavorable al primero $\mathrm{y}$, lo más importante, impide que sean comparables sobre esta dimensión.

El Gasto Público en educación en 1998 alcanzó al 3.9\% del PIB, incluyendo el aporte del Ministerio, el gasto de las Municipalidades y la inversión educacional efectuada por Fondo Nacional de Desarrollo Regional, que se dirige a los establecimientos subvencionados (municipales y privados). Por su parte, el Gasto Privado en Educación para ese año se estima en un 2.9\% del PIB, cuya distribución favorece a los sectores de mayores ingresos (Mineduc, 2001a).

De esta forma podemos señalar que la comparación de los establecimientos por grupo socioeconómico y dependencia del establecimiento omite un factor de inequidad

4. De paso revisar la multicolinealidad entre las variables y determinar los factores reales que se están midiendo

5. Ello en función de las diferencias de ingresos económicos entre los diversos quintiles determinados en la Encuesta Casen (Caracterización Socioeconómica de las familias). 
que tiene incidencia sobre los resultados escolares: el nivel de equipamiento e infraestructura escolar, partiendo de una situación ficticia (igualdad de procesos básicos) que favorece a los establecimientos mejor dotados, que suelen ser los que incorporan financiamiento de los padres.

La debilidad anotada pone en duda la segunda de las hipótesis explicativas del modelo: que las diferencias "intragrupo" se deben a las variables de "gestión escolar". Puede ser que respondan a esta situación u otra, o bien a una combinación de ellas. Sin embargo no es posible establecerlo dado que no han sido aisladas debidamente las variables para poder identificar estos efectos. Por ende, las conjeturas que se deriven de los diferenciales de resultados en establecimientos de condiciones socioeconómicas similares no permiten seriamente su atribución al campo de la gestión.

\section{Resultados según dependencia y grupo socioeconómico}

\section{Resultados según dependencia de los establecimientos}

El análisis de la Tabla $n^{\circ} 4$ muestra que la población de establecimientos escolares municipales es fundamentalmente de nivel bajo y medio bajo (7.7 de cada 10), seguida de los de nivel medio (1.98) y una ínfima presencia de nivel medio alto.

El grupo Particular Subvencionado se concentra en proporciones bastante similares en la población Baja y Medio Baja (casi el $30 \%$ ), con mayor presencia en el sector medio
(3.8 de cada 10) y con un importante grupo en el Medio Alto (2.8 de cada 10) y casi desaparecen en la población de nivel Alto.

Los establecimientos Particulares Pagados se distribuyen 2 de cada 10 en el nivel Medio Alto, y 8 de 10 en el Alto.

\section{Resultados por pruebas según características socioeconómicas del establecimiento}

Los resultados alcanzados por los establecimientos en las distintas pruebas están estrechamente correlacionados, tanto en términos totales, como segmentado el universo según agrupación socioeconómica o dependencia del establecimiento (Tabla $n^{\circ}$ 5).

Los valores expuestos muestran una estrecha, poderosa y significativa asociación entre los resultados de las distintas pruebas. ldénticas tendencias se encontraron en los análisis por cada uno de los cinco grupos socioeconómicos y por la variable dependencia. En todos los estratos generados los resultados alcanzados mantienen esta lógica: alta significación estadística y estructuras muy fuertes de relación positiva.

Dada la estrecha y fuerte asociación encontrada entre los resultados de las distintas pruebas y las estructuras de análisis: grupos socioeconómicos y dependencia, se opta por continuar el análisis con las pruebas de matemática y castellano (pese a su estrecha relación), pues se entiende que sus resultados serán extensivos a las otras dos pruebas, cualquiera que sea la segmentación del universo que se realice.

Tabla $n^{\circ}$ 4. Distribución de los establecimientos por dependencia y grupo socioeconómico

\begin{tabular}{|c|c|c|c|c|c|c|}
\hline Dependencia & Bajo & Medio Bajo & Medio & Medio Alto & Alto & Total \\
\hline Municipal & 847 & 900 & 299 & 43 & . & 2089 \\
\hline Corporación Municipal & 113 & 358 & 267 & 42 & -. & 780 \\
\hline Corp.Municipal con financiamiento compartida & 1 & 2 & 3 & 4 & 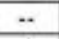 & 10 \\
\hline Particular subvencionado & 196 & 203 & 203 & 42 & 4 & 648 \\
\hline Part. subvencionado con financiamiento compartido & 3 & 73 & 454 & 410 & 13 & 953 \\
\hline Corporativizado & $\cdots$ & 1 & $-\cdot$ & 1 & 1 & 3 \\
\hline Particular pagado & $*$ & 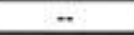 & $m$ & 105 & 445 & 550 \\
\hline
\end{tabular}


Concomitantemente, una relación tan estrecha entre los resultados lleva a revisar la significación de aplicar cuatro pruebas, que por su fuerte asociación, dan a entender que comparten muchos elementos de la medición, es decir podrían estar midiendo un factor, ${ }^{6}$ o bien los procesos mentales asociados a los contenidos son muy similares. En este caso, por tratarse de contenidos, puede significar que se mide una habilidad o forma de estudiar común a las pruebas $^{7} \mathrm{y}$, por consiguiente, formas de enseñar.

Para efectos de determinar si las estructuras de dependencia de los establecimientos y los criterios de clasificación socioeconómica presentan asociación con los resultados alcanzados, se llevaron a cabo procedimientos de análisis de varianza.

La estructura original de agrupación socioeconómica (cinco grupos) da cuenta de resultados de significación. Sin embargo, a la luz de la estructura de promedios que se advierte en la Tabla $n^{\circ} 7$, donde los sectores de menores ingresos también tienen los menores resultados, se propone realizar la siguiente agrupación de establecimientos buscando asociarlos directamente a

6. El análisis factorial de las cuatro series de puntajes arroja un único factor con un $92 . \%$ de varianza compartida.

7. Los residuos en la regresión muestran que se trata de resultados estructurales: en todas las pruebas se distribuye normalmente.

Tabla $\mathbf{n}^{*}$ 5. Natriz de correlación (Pearson) resultados puntajes pruebas del Universo.

\begin{tabular}{|c|c|c|c|}
\hline & $\begin{array}{l}\text { Puntaje } \\
\text { Castellano }\end{array}$ & $\begin{array}{l}\text { Puntaje } \\
\text { Historia }\end{array}$ & $\begin{array}{c}\text { Puntaje Cs. } \\
\text { Naturales }\end{array}$ \\
\hline Puntaje Natemsitica & $910 \times 1$ & $\left..885{ }^{* 01}\right)$ & $.865[-0]$ \\
\hline Puntaje Castellano & & $\left.9090^{\circ+\infty}\right)$ & 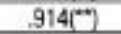 \\
\hline Puntaje Historia & & & $.899\left(^{\circ+1}\right)$ \\
\hline
\end{tabular}

T+7) signilicative exn $p<001$

Tabla $n^{*} 6$. Análisis de varianza de los puntajes en matemática y castellano según grupos socioeconómicos

\begin{tabular}{|l|l|r|r|r|r|r|}
\hline \multicolumn{2}{|c|}{} & \multicolumn{1}{c|}{$\begin{array}{c}\text { Suma de } \\
\text { cuadrados }\end{array}$} & \multicolumn{1}{c|}{ gl } & \multicolumn{1}{c|}{$\begin{array}{c}\text { Media } \\
\text { cuadrattica }\end{array}$} & \multicolumn{1}{c|}{ F } & Sig. \\
\hline \multirow{2}{*}{$\begin{array}{l}\text { Puntaje } \\
\text { matemática }\end{array}$} & Inter-grupos & 1972921.275 & 4 & 493230.319 & 1168.396 & .000 \\
\cline { 2 - 8 } & Intra-grupos & 2126775.160 & 5038 & 422.147 & & \\
\cline { 2 - 8 } & Total & 4099696.435 & 5042 & & & \\
\hline \multirow{2}{*}{$\begin{array}{l}\text { Puntaje } \\
\text { Castellano }\end{array}$} & Inter-grupos & 1907882.754 & 4 & 476970.689 & 1320.696 & .000 \\
\cline { 2 - 8 } & Intra-grupos & 1819200.299 & 5038 & 361.096 & & \\
\cline { 2 - 8 } & Total & 3727003.053 & 5042 & & & \\
\hline
\end{tabular}

Tabla $n^{*} 7$. Promedios en matemática y castellano según nivel socioeconómico

\begin{tabular}{|c|c|c|c|c|c|c|}
\hline \multicolumn{2}{|c|}{ NSE } & \multirow{2}{*}{$\frac{N}{1160}$} & \multirow{2}{*}{$\begin{array}{r}\text { Media } \\
229,87 \\
\end{array}$} & \multirow{2}{*}{$\begin{array}{c}\begin{array}{c}\text { Desv. } \\
\text { estándar }\end{array} \\
21.04 \\
\end{array}$} & \multirow{2}{*}{$\begin{array}{r}\text { Minimo } \\
169\end{array}$} & \multirow{2}{*}{$\begin{array}{r}\text { Máximo } \\
31\end{array}$} \\
\hline \multirow{6}{*}{$\begin{array}{l}\text { Puntaje } \\
\text { Matemática }\end{array}$} & Bajo & & & & & \\
\hline & Medio bajo & 1539 & 232.19 & 16.41 & 171 & 311 \\
\hline & Medio & 1230 & 247.32 & 19.32 & 168 & 314 \\
\hline & \begin{tabular}{|l|} 
Medio alto \\
\end{tabular} & 653 & 272.41 & 2253 & 181 & 350 \\
\hline & Allo & 462 & 290.64 & 29.82 & 165 & 352 \\
\hline & Total & 5043 & 245.91 & 28.52 & 165 & 352 \\
\hline \multirow{6}{*}{$\begin{array}{l}\text { Puntaje } \\
\text { castellano }\end{array}$} & Bajo & 1160 & 228.16 & 18.98 & 170 & 300 \\
\hline & Medio bajo & 1538 & 232.37 & 15.55 & 177 & 324 \\
\hline & Medio & 1230 & 248.28 & 17.96 & 170 & 322 \\
\hline & Medio alto & 653 & 272.56 & 20.88 & 183 & 343 \\
\hline & Alto & 462 & 287.28 & 27.54 & 170 & 338 \\
\hline & Total & 5043 & 245.52 & 27.19 & 170 & 343 \\
\hline
\end{tabular}


dependencia, sin consideración el tipo o grado de financiamiento que tengan.

\section{Resultados agrupando los establecimientos en tres categorias de dependencia}

La población contempla ocho categorías iniciales de dependencia. Con la finalidad de reducir el efecto de grupos muy pequeños de establecimientos $\mathrm{y}$, a la vez, para maximizar el poder discriminatorio, se procesaron los datos utilizando una reagrupación en 5 grupos por Dependencia y luego en 3 grupos de acuerdo a la especificación anterior. La comparación de los resultados, especialmente por vía del análisis discriminante y eficiencia de la clasificación, permiten adoptar un modelo de tres grupos de Dependencia, marcados por el origen del financiamiento más que por la naturaleza de los dueños.

Se hicieron dos análisis de los resultados en las pruebas de Matemáticas y Castellano. El primero, una regresión múltiple con las pruebas como dependientes y Dependencia y Grupo Socioeconómico como independientes. ${ }^{8}$ Los resultados observados en la Tabla $n^{\circ} 8$ ponen en evidencia el peso del GSE por sobre la
Dependencia, con lo cual se confirma la apreciación inicial de que las diferencias en los resultados en las pruebas Simce tienen su origen en las diferencias de nivel socioeconómico de los niños más que en la propiedad o dependencia del establecimiento en que se educan. Los datos de la Tabla $n^{\circ} 8$ muestran que la primera variable que entra en la explicación del puntaje en Matemática es Grupo Socioeconómico, con un 43.6\% de varianza explicada; la Dependencia entra en segundo lugar con un $0.5 \%$ de varianza explicada adicional. La Tabla $n^{\circ} 9$ muestra los resultados del análisis de la varianza sobre la regresión, donde ambos modelos explican significativamente; sin embargo, considerando el principio de parsimonia, es preferible el modelo 1 que sólo considera al Nivel Socioeconómico como variable predictora y deja fuera de ese rol a la Dependencia del establecimiento.

Los procesamientos para el caso de

8. Se procesaron los datos en el modelo de regresión por stepwise incorporando además la variable "tamaño", indicada por el número de alumnos que rindieron las pruebas. Sin embargo, el aporte de esta variable a la explicación es completamente despreciable ( $0.1 \%$ de varianza adicional). Puede hipotetizarse que el tamaño del establecimiento también se relaciona con Grupo Socioeconómico, especialmente considerando al hecho de que las escuelas más pequeñas son precisamente las municipales, que atienden a los sectores más vulnerables, que son los alumnos rurales.

Tabla $n^{\circ}$ 8. Resumen de la Regresión con Matemática como dependiente.

\begin{tabular}{|l|l|c|c|c|}
\hline \multicolumn{1}{|c|}{ Modelo } & R & $\mathbf{R}^{\mathbf{2}}$ & $\mathbf{R}^{2}$ ajustado & Error estándar de la estima \\
\hline 1 (a) & $.660(\mathrm{a})$ & 436 & 436 & 21.41 \\
\hline 2 (b) & $664(\mathrm{~b})$ & 441 & 441 & 21.32 \\
\hline
\end{tabular}
(a) Predictores: (Constante), Grupo sociseconómico
(b) Predictores: (Constarte), Grupo sociceoonómico, Dependencia maximzada

Tabla $n^{\circ}$ 9. ANOVA de la Regresión con Matemática corno dependiente.

\begin{tabular}{|c|c|c|c|c|c|c|}
\hline & Modelo & $\begin{array}{c}\text { Suma de } \\
\text { cuadrados }\end{array}$ & gl & $\begin{array}{c}\text { Cuadrado } \\
\text { medio }\end{array}$ & $\mathbf{F}$ & Sig. \\
\hline \multirow{3}{*}{1 (a) } & Pegresión & 1788106.528 & 1 & 1788106.528 & 3899.414 & .000 \\
\hline & Pesidual & 2311589.908 & 5041 & 458.558 & & \\
\hline & Total & 4099696.435 & 5042 & & & \\
\hline \multirow{3}{*}{2 (b) } & Pegresión & 1808970.291 & 2 & 904435.146 & 1990.026 & .000 \\
\hline & Residual & 2290726.144 & 5040 & 454.509 & & \\
\hline & Total & 4099696.435 & 5042 & & & \\
\hline
\end{tabular}


los resultados en Castellano dan valores absolutamente homólogos a los de matemáticas.

\section{Resultados según grupo socioeconómico en cada una de las categorías de dependencia.}

A fin de establecer con mayor precisión el peso de la variable socioeconómica, se evaluaron los puntajes al interior de cada uno de los grupos de dependencia. Los resultados (ver Tablas $n^{\circ}$ s $10,11,12$ ) indican que en los tres grupos de Dependencia hay diferencias significativas de los promedios en su interior, donde los resultados diferenciales obedecen linealmente al nivel socioeconómico de la familia.

En consecuencia, se observa con evidencia la estructuración de los puntajes en función de los criterios socioeconómicos, confirmando el conjunto de planteamientos que se han señalado. Por ello es inapropiado e incorrecto afirmar que los menores resultados observados en las escuelas de dependencia municipal obedecen al hecho de que son escuelas públicas y no privadas. Lo correcto es afirmar que las escuelas municipales tienen inferiores resultados porque atienden a los hijos de los más pobres del país. Esto se ve reforzado más adelante por el análisis de los costos asociados a cada una de las modalidades de dependencia.

Atendiendo a los antecedentes expuestos en la sección, ciertamente el factor socioeconómico es un determinante fundamental de los resultados escolares. Si bien ello ocurre como máxima en todas las sociedades (Peck, 2001; Jenkss, 1972), el impacto del sistema como un elemento compensador del factor socioeconómico no logra en Chile los estándares que alcanzan estas variables, por

Tabla $n^{\circ}$ 10. ANOVA de Puntaje matemática sobre nivel socioeconómico, colegios de dependencia municipal

\begin{tabular}{|l|r|r|r|r|r|}
\hline \multicolumn{1}{|c|}{ Fuente } & \multicolumn{1}{|c|}{$\begin{array}{c}\text { Type III Sum of } \\
\text { Squares }\end{array}$} & \multicolumn{1}{c|}{ df } & Mean Square & \multicolumn{1}{c|}{ F } & Sig. \\
\hline hiterceat & 68334839.860 & 1 & 63334339.860 & 208889.788 & .000 \\
\hline GSE & 200688.141 & 3 & 66896.047 & 204.492 & .000 \\
\hline Error & 944107.177 & 2886 & 327.133 & & \\
\hline Total & 162035915.000 & 2890 & & & \\
\hline
\end{tabular}

Tabla $n^{\circ}$ 11. ANOVA de Puntaje matemática sobre Nivel Socioeconómico, Colegios de Dependencia Particular Subvencionada

\begin{tabular}{|l|r|r|r|r|r|}
\hline \multicolumn{1}{|c|}{ Source } & \multicolumn{1}{|c|}{$\begin{array}{c}\text { Type Ill Sum of } \\
\text { Squares }\end{array}$} & df & Mean Square & \multicolumn{1}{c|}{ F } & Sig. \\
\hline Intercapt & 22775555.724 & 1 & 22775555.724 & 53477.602 & .000 \\
\hline GSE & 554927.441 & 4 & 139731.860 & 325.746 & .000 \\
\hline Error & 679719.835 & 1596 & 425.890 & & \\
\hline Total & 101228659.000 & 1601 & & & \\
\hline
\end{tabular}

Tabla $n^{\circ}$ 12. ANOVA de Puntaje matemática sobre Nivel Socioeconómico, Colegios de Dependencia Particular Pagada

\begin{tabular}{|l|r|r|r|r|r|}
\hline \multicolumn{1}{|c|}{ Source } & \multicolumn{1}{|c|}{$\begin{array}{c}\text { Type III Sum of } \\
\text { Squares }\end{array}$} & \multicolumn{1}{c|}{ df } & Mean Square & F & Sig. \\
\hline $\begin{array}{l}\text { Corrected } \\
\text { Model }\end{array}$ & $554927.441(\mathrm{a})$ & 4 & 138731.860 & 325.746 & .000 \\
\hline intercept & 22775555.724 & 1 & 22775555.724 & 53477.602 & .000 \\
\hline GSE & 554927.441 & 4 & 138731.860 & 325.746 & .000 \\
\hline Errar & 679719.835 & 1596 & 425.890 & & \\
\hline Total & 101228659.000 & 1601 & & & \\
\hline
\end{tabular}


ejemplo, en los países asiáticos. En el país, las diferencias sociales son con demasiada fuerza, también, generadoras de desigualdades educativas importantes, y allí es donde radica la gravedad del problema. Si las diferencias fueran menores y además estuvieran estructuradas a partir de pisos en los cuales el que se encuentra en el nivel inferior queda habilitado para determinados estándares, esta situación podría verse con una mirada más positiva, pero no es el caso nuestro. Las diferencias son significativas y se explican en demasía por las variables socioeconómicas.

En segundo lugar, los hallazgos muestran que en general la temática de la gestión está subordinada definitivamente al factor socioeconómico. En consecuencia, los tres segmentos estructurados confirman que no es del todo relevante la "gestión escolar" en la explicación, si bien es completamente admisible que existan casos excepcionales donde una excelente gestión lleve a logros que sus pares no alcanzan.

Finalmente, podríamos pensar que estos resultados no son aún más diferenciados gracias a los aspectos compensatorios que tiene el sistema público, incluyendo en este marco la jornada escolar completa en desarrollo.

En la sección siguiente se analiza someramente el costo de estos puntajes y la valorización de las diferencias entre cada grupo.

\section{El valor económico de las diferencias}

Esta sección tiene por finalidad evaluar económicamente las diferencias de logro. Para ello, se generaron tres grupos de dependencia apareada por criterio de financiamiento: (1) Establecimientos que sólo reciben subvención del Estado (sean municipales o particulares); (2) establecimientos que además de la subvención estatal tienen financiamiento compartido, y (3) establecimientos particulares pagados sin subvención del Estado.

El análisis se realiza sólo con los resultados de la prueba de matemáticas dado que el ejercicio con la de castellano reportaría datos muy similares. Para efectos de lo realizado se asume que:

- El valor de las colegiaturas o subsidios mensuales promedio de cada grupo corresponde al costo directo real unitario/mes por estudiante.

- Por ende, se consideran equivalentes las escuelas en términos de infraestructura, equipamiento, etc., en función de la inaccesibilidad de estos datos.

- lgualmente no se incluirá el gasto privado en educación de cada sector como cuotas de incorporación, matrícula, etc. Se sabe (Casen, 2000) que esta variable se mueve paralelamente al nivel socio-económico.

- Se trabajará con un aporte de diez valores mensuales por año.

Entre los factores condicionantes, sabemos igualmente que no existe el costo cero en educación. Un establecimiento escolar subvencionado, cualesquiera que sean sus resultados, recibe el valor de la subvención, aquel que tenga buenos logros puede ser beneficiado con

Tabla $\mathrm{N}^{\circ}$ 13. Valores de las distribuciones de las pruebas para cada estrato o grupo

\begin{tabular}{|l|r|r|r|r|r|r|}
\hline & \multicolumn{2}{|c|}{ Grupo sólo Subvención } & \multicolumn{2}{c|}{ Grupo Subvención + Pago } & \multicolumn{2}{c|}{ Grupo sólo Pago } \\
\hline & Matemática & \multicolumn{1}{|c|}{ Castellano } & Matemática & Castellano & Matemáltica & Castallano \\
\hline Media & 235.96 & 235.54 & 258.86 & 259.61 & 286.65 & 284.38 \\
\hline N & 3517 & 3517 & 974 & 974 & 549 & 549 \\
\hline Desv. tip. & 20.95 & 19.83 & 25.60 & 23.44 & 30.02 & 28.07 \\
\hline Mediana & 234.00 & 234.00 & 257.00 & 260.00 & 292.00 & 290.00 \\
\hline Rango & 181 & 173 & 158 & 154 & 187 & 168 \\
\hline Minimo & 169 & 170 & 168 & 170 & 165 & 170 \\
\hline Máximo & 350 & 343 & 326 & 324 & 352 & 338 \\
\hline
\end{tabular}


aportes adicionales que, por lo general, son marginales. El piso para cada sector se ha definido de acuerdo a los valores de subvención que rigen a la fecha, y a estimaciones de los cobros promedio de los establecimientos con financiamiento compartido y particular pagados producto de un estudio muestral.

El piso es la Subvención de educación básica de US\$43 por mes por alumno. ${ }^{9}$

El piso de los establecimientos con financiamiento compartido es de US\$45+ US $\$ 31^{10}=$ US $\$ 76$. US\$185."

El piso del particular pagado es de

Sin necesidad de un análisis sofisticado, atendiendo a los valores mensuales definidos, podríamos decir que los establecimientos que funcionan sólo con subvención muestran resultados importantes en esta materia. El estándar de logro de los establecimientos con sólo subvención estatal es un 9.7\% inferior al de los establecimientos subvencionados con pago, mas el valor mensual de éstos es un 73\% superior.

El estándar de logro de los particulares respecto de los subvencionados es un $22 \%$ superior, su contraparte económica es un 330\% más elevada.

El estándar de logro de los particulares pagados es un $11 \%$ superior a los de financiamiento compartido pero su valor mensual promedio es un 143\% más elevado.

Si los resultados escolares pudieran explicarse linealmente, no cabría duda razonable. El sistema con subvención sería claramente más eficiente. Pero sabemos que ello no es así. Sin embargo, no deja de ser un factor ilustrativo de magnitud pensar que si se quiere alcanzar el nivel de los particulares, debería al menos agregarse el 330\% más de recursos, lo que parece insostenible para las finanzas públicas.

Por su parte, cada uno de los 51 puntos de diferencia que median a favor de los establecimientos particulares pagados implican un costo anual de US\$28. Es decir, el diferen- cial de inversión entre un establecimiento subvencionado y uno particular pagado es de US\$1.428 por año. En consecuencia, si asumimos que el SiMce de los $8^{\text {os }}$ años considera la inversión de 4 años ( $5^{\circ}$ a $8^{\circ}$ ), los establecimientos pagados han tenido los estándares superiores a razón de una inversión unitaria adicional directa por alumno de US\$5.712, ${ }^{12}$ por sobre la inversión base de US\$1.720 (correspondientes a la subvención estatal sin aportes adicionales).

La situación de los establecimientos con financiamiento compartido muestra que los 23 puntos adicionales promedio que obtienen tienen un costo anual por punto/alumno de US\$1,35. El diferencial de inversión US\$31 al año, y de US\$124 para el período de cuatro años.

Los aspectos mencionados nos llevan a pensar que es muy difícil que la educación pública pueda romper el círculo de la pobreza con estos estándares de inversión. Mejorar la gestión escolar es importante, pero incrementar el volumen de recursos es mayormente relevante en aquellos grupos que requieren compensar carencias tan significativas.

El actual sistema de financiamiento de la educación pública ha generado un desplazamiento de la población del sector municipal al particular subvencionado y en lo fundamental al segmento del financiamiento compartido (Hsieh; Urdiola, 2002), de forma que los incrementos de aprendizaje de este sector, obedecen más al mejoramiento del nivel socioeconómico promedio que a un efectivo y superior tratamiento pedagógico asociado a la dependencia privada del establecimiento.

En este marco, abogar por la privatización de la educación parece no ser más

\footnotetext{
9. Estimando un valor referencial del dólar a $\$ 650$.

10 Estimado a partir de un estudio muestral de los cánones cobrados. 11. Id a anterior.

12. Como se señalara anteriormente, el diferencial implica el financiamiento adicional de 3.3 niños en un establecimiento escolar subvencionado. Dicho en otros términos, por cada costo de estudiante particular se financia 4.3 estudiantes en el sistema subvencionado.
} 
que una actitud ideológica. La dependencia del establecimiento es irrelevante ante las condiciones de pobreza de la población. Mayor relevancia debería tener la implementación de un sistema diferenciado de subvención escolar (Donoso; Schmal, 2002) que provea los recursos para reducir drásticamente la influencia del factor socioeconómico familiar sobre los resultados escolares.

\section{Consideraciones finales}

El artículo sostiene que los resultados del último Simce aplicado a los $8^{\circ \mathrm{os}}$ años básicos del país (año 2000), ratifican la alta incidencia que tienen las desigualdades sociales sobre las desigualdades educativas.

Los esfuerzos realizados en el país por reducir el impacto del primero sobre el segundo elemento no muestran frutos significativos para todos los estratos sociales. De esta forma, la determinante socioeconómica gravita en demasía sobre el Simce. Ello se comprueba analizados los datos por la clasificación socioeconómica original del Ministerio, como también por las agrupaciones que se generan a partir de la relación dependencia del establecimiento y aportes económicos. Por lo general se van a asociar positivamente según se incremente el nivel socioeconómico lo hará el de logros.

Si bien esta relación ha sido documentada en diferentes latitudes desde hace más de 30 años, las pruebas aplicadas en Chile muestran lo lejos que se está de superar, neutralizar o mitigar este abismo, pese a los esfuerzos realizados por dotar a la educación pública de equipamiento e infraestructura, asi como de perfeccionamiento a los docentes. Se puede decir que, paralelamente, el sistema de financiamiento de la educación ha contribuido a segregar aún más los resultados escolares, toda vez que los estímulos financieros fundamentales se reducen a captar "buenos" estudiantes (asumidos como los más iguales o con menor costo agregado o con mayor capacidad de pago) y no a obtener y premiar los buenos resultados académicos.

En lo fundamental se ha producido un éxodo de alumnos del sistema público al subvencionado con financiamiento compartido que ratifica la segregación social e incide sobre el alza de logros de este sector. Ello se observa en los datos consignados. El grupo que funciona con la subvención estatal tiene siempre resultados escolares menores que los que alcanza aquel que tiene financiamiento compartido (o aportes de los padres) como también del particular pagado. Sin embargo, las diferencias de registro positivo muestran también costos muy superiores que no se condicen directamente entre el diferencial alcanzado en logros con el que deben realizar para financiarlo.

En este marco pueden destacarse dos aspectos. Uno que para el sector más pobre de la población los establecimientos municipales son eficientes, y en segundo lugar, que en los niveles socioeconómicos en que hay plena competencia (donde el mercado funciona) la dependencia del establecimiento es secundaria, siendo el nivel socioeconómico el factor explicativo dominante.

Para efectos de poder realizar un análisis más preciso, debería generarse un índice socioeconómico que permitiera su asociación con los logros y así poder estructurar con mayor precisión los análisis de manera que las diferencias establecidas representen un factor real de comparación a partir de cual podrían reducirse los resultados a unidades iguales. En razón de ello, los resultados actuales ocultan una serie de situaciones que no es posible determinar más que a nivel macro y que en lo fundamental subestiman el efecto de las variables socioeconómicas.

Por otra parte, es importante destacar que la comparación de los establecimientos por grupo socioeconómico y dependencia del establecimiento omite un factor de inequidad que tiene incidencia sobre los resultados escolares: el nivel de equipamiento e infraestructura escolar, partiendo de una situación ficticia 
(igualdad de procesos básicos) que favorece a los establecimientos mejor dotados, que suelen ser los que incorporan financiamiento de los padres. La debilidad anotada pone en duda la segunda de las hipótesis explicativas del modelo: que las diferencias "intragrupo" se deben a las variables de "gestión escolar". Puede ser que respondan a esta situación en algunos casos específicos y excepcionales, pero la evidencia es contundente al incorporar el nivel socioeconómico como variable explicativa.

Finalmente, analizando el tema financiero, bajo un conjunto de supuestos debidamente detallados, se aprecia que existe mucha asimetría entre los estándares de resultados registrados y el costo de estos. Esta situación sugiere varias dudas: ¿Qué nivel de inversión debería asignarse a la educación pública para enfrentar adecuadamente la tarea? ¿es viable la actual tasa de inversión en educación pública con los objetivos de país que se buscan? ¿cómo estructurar debidamente un sistema de financiamiento de la educación que pueda romper el círculo de la pobreza?

En razón de lo señalado, más que preguntarnos por qué los establecimientos subvencionados (sean municipales o particulares) tienen bajos rendimientos, la afirmación es por qué en nuestro país ser pobre determina bajos resultados escolares. Más que avergonzarse por los bajos resultados escolares, es más pertinente hacerlo por los altos niveles de pobreza de nuestra población, que son los que inciden en esa situación. ${ }^{13}$

13. Incluso podría argumentarse que el nivel socioeconómico promedio de la población de $8^{\circ}$ año básico es superior al de los $4^{0 \mathrm{~s}}$ años básicos, de forma que en la estructura socioeconómica debería ser más fuerte como agente explicativo.

\section{Referencias bibliográficas}

AEDO, Cristián; SAPELLI, Claudio. El sistema de vouchers en educación: una revisión de la teoría y evidencia empírica para Chile. Estudios Públicos, n. 82, p. 35-82, 2001.

ALVARIÑO, Celia; ARZOLA, S.; BRUNNER, J.J.; RECART, M. 0.; VIZCARRA, R. Gestión Escolar: un estado del arte de la literatura. Paideia, Concepción, n. 29, 2001.

BEYER, Harold. Educación y desigualdad de ingresos. Estudios Públicos, n. 77, p. 97-130, 2000.

BRAVO, David; MANZI, Jorge. Equidad y resultados educacionales: SIMCE y PAA. Santiago: Proyecto SIES, 2002.

BRITTON, Edward; RAIZEN, Senta. Examining the examinations: an international comparaison of science and mathematics examinations for College-Bound Students. Boston: Kluwer Academic Publishers, 1996.

CEPAL-Unesco. Educación y conocimiento: eje de la transformación productiva con equidad. Santiago, CL: Naciones Unidas, 1992.

COLEMAN J. S. Equality en achievement in education. San Francisco: Westview press; Boulder, 1990.

Equal educational opportunity. [s.I.]: Harvard University Press; Cambridge Mass, 1969.

COMISIÓN Nacional para la Modernización de la Educación. Informe de la Comisión Nacional para la Modernización de la Educación. Santiago: Editorial Universitaria, 1995.

DONOSO, Sebastián; SCHMAL, Rodolfo. Nuevos criterios para el cálculo del financiamiento de la educación pública en Chile. In: CONGRESO LATINOAMERICANO DE ADMINISTRACIÓN EDUCACIONAL, 5., 2002, Santiago.

EYZAGUIRRE, Bárbara; LE FOULON, Carmen. La calidad de la educación chilena en Cifras. Estudios Públicos, n. 84, p. 87-204, 2001.

FONTAINE, Arturo. Equidad y educación: cinco proposiciones. CEP, Santiago, n.255, ene. 2002. 
HSEIH, Chang T.; URQUIOLA, Miguel. When school compete, how do the compete? An assessment of Chile's nationwide school voucher program. 2002. World Bank's Development Research Group.

JENCKS, C. et al. Inequality. New York: Basic Books, 1972.

MINEDUC. Antecedentes y resultados del SIMCE 2000 publicados en la web respectiva. Educacional MINEDUC (2001a) Sistema Escolar. Disponível em: <http: www.mineduc.cl/sistemaeducacional/estadisticas/estadisticas.html>. Acesso em: 2001.

PECK, Bob. The poor stay poor and the rich stay rich. Issues in Educational Research, v. 11, 2001.

Recebido em 06.06.02

Aprovado em 29.08.02

Sebastián Donoso Díaz es profesor investigador del Instituto de Investigación y Desarrollo Educacional de la Universidad de Talca y profesor del área de Política Educacional del Magíster en Política y Gestión Educacional de la misma Universidad.

Gustavo Hawes Barrios es profesor investigador del Instituto de Investigación y Desarrollo Educacional de la Universidad de Talca y profesor del área de Epistemología Aplicada del Magíster en Política y Gestión Educacional de la misma Universidad. 